CONGENITAL HEART DISEASE

\title{
Magnetic resonance imaging guided catheterisation for assessment of pulmonary vascular resistance: in vivo validation and clinical application in patients with pulmonary hypertension
}

\author{
T Kuehne, S Yilmaz, I Schulze-Neick, E Wellnhofer, P Ewert, E Nagel, P Lange
}

Heart 2005;91:1064-1069. doi: 10.1136/hrt.2004.038265

See end of article for authors' affiliations

Correspondence to: Dr Titus Kuehne, Department of Congenital Heart Diseases and Paediatric Cardiology, German Heart Institute Augustenburger Platz 1, Berlin 13353, Germany; kuehne@dhzb.de

21 October 2004

\begin{abstract}
Objectives: To validate in vivo a magnetic resonance imaging (MRI) method for measurement of pulmonary vascular resistance (PVR) and subsequently to apply this technique to patients with pulmonary hypertension (PHT).

Methods and results: PVR was assessed from velocity encoded cine MRI derived pulmonary artery (PA) flow volumes and simultaneously determined invasive PA pressures. For pressure measurements flow directed catheters were guided under magnetic resonance fluoroscopy at 1.5 T into the PA. In preliminary validation studies (eight swine) PVR was determined with the thermodilution technique and compared with PVR obtained by MRI (0.9 (0.5) $\vee 1.1(0.3)$ Wood units $\left.\cdot \mathrm{m}^{2}, \mathrm{p}=0.7\right)$. Bland-Altman test showed agreement between both methods. Inter-examination variability was high for thermodilution $(6.2(2.2) \%)$ but low for MRI measurements (2.1 (0.3)\%). After validation, the MRI method was applied in 10 patients with PHT and five controls. In patients with PHT PVR was measured at baseline and during inhalation of nitric oxide. Compared with the control group, PVR was significantly increased in the PHT group (1.2 (0.8) $v 13.1$ (5.6) Wood units $\cdot \mathrm{m}^{2}, \mathrm{p}<0.001$ ) but decreased significantly to $10.3(4.6)$ Wood units $\cdot \mathrm{m}^{2}$ during inhalation of nitric oxide $(p<0.05)$. Inter-examination variability of MRI derived PVR measurements was $2.6(0.6) \%$. In all experiments (in vivo and clinical) flow directed catheters were guided successfully into the PA under MRI control.

Conclusions: Guidance of flow directed catheters into the PA is feasible under MRI control. PVR can be determined with high measurement precision with the proposed MRI technique, which is a promising tool to assess PVR in the clinical setting.
\end{abstract}

$\mathrm{P}$ ulmonary hypertension (PHT) is an important cause of cardiological morbidity and mortality and is a major factor complicating the management of children with congenital heart disease. ${ }^{12}$ In patients with delayed primary intracardiac cardiac repair, PHT may result. In these patients, as well as in patients who have primary forms of the disease, pulmonary vasodilatation may be achieved with selective pulmonary vasodilators. ${ }^{3}$ However, clinicians caring for such patients are confronted with limited approaches for measurement of pulmonary vascular resistance (PVR).

The thermodilution technique and the Fick method of deriving PVR are susceptible to several sources of error, particularly in the presence of intracardiac shunts or right ventricular dysfunction..$^{4-7}$ Doppler guidewire measurements reflect only a small region of the pulmonary vasculature, which does not necessarily account for inhomogeneous pulmonary perfusion. ${ }^{8-10}$

Recent improvements in magnetic resonance imaging (MRI) hardware and software allow for MRI guided cardiac catheterisation and have enabled invasive measurement of pulmonary artery (PA) pressure and the conductance of catheter based intervention in animals and the first human studies. ${ }^{11-13}$ In addition, velocity encoded cine (VEC) MRI is widely accepted as an accurate and reproducible method for quantification of PA flow volumes and pulmonary perfusion. ${ }^{14-17}$ Muthurangu and colleagues ${ }^{13}$ recently reported the combination of VEC MRI derived PA flow volumes and invasively measured PA pressures for assessment of PVR in patients without or with mild PHT.
The aims of our study were, firstly, to validate the proposed MRI method in vivo and, secondly, to apply this technique in the clinical setting to patients with a broad range of PVR.

\section{PATIENTS AND METHODS}

Validation study

Eight swine (mean (SD) weight 19 (4) kg) were studied. All procedures were performed in accordance with the National Institutes of Health guidelines for care and use of laboratory animals and with the approval of the Committee of Animal Research at our institution. For procedures, the animals were given $1.5 \%$ isoflurane inhalation for maintenance of general anaesthesia. After completion of the study, the animals were euthanised with sodium pentobarbital $(200 \mathrm{mg} / \mathrm{kg}$ intravenously).

Vascular access was gained by the Seldinger technique in the femoral vein and artery. Left atrial (LA) pressures were measured with a 6 French liquid filled wedge catheter from the arterial side. A 6 French thermodilution catheter was then advanced through the femoral venous sheath under $x$ ray fluoroscopy and the distal port was positioned in the main PA. PA pressures were measured through the liquid filled catheter. Right ventricular thermodilution cardiac outputs were computed after injection of $10 \mathrm{ml}$ of $10^{\circ} \mathrm{C}$ cold isotonic saline solution through the proximal port of the catheter. On completion of these measurements all catheters

Abbreviations: LA, left atrial; MRI, magnetic resonance imaging; PA, pulmonary artery; PHT, pulmonary hypertension; PVR, pulmonary vascular resistance; VEC, velocity encoded cine 
were removed from the vascular system and the animals were transferred into a $1.5 \mathrm{~T}$ MRI unit (Intera CV, Philips Medical Systems, Best, the Netherlands) located about $30 \mathrm{~m}$ from the catheterisation laboratory. A 6 French wedge catheter was inserted through the femoral vein sheath and guided under magnetic resonance fluoroscopy into the PA. For guidance the balloon of the wedge catheter was inflated with carbon dioxide. Images were viewed on line on an inroom monitor (Philips Medical Systems). LA pressures were not measured again during MRI. Vital signs continuously monitored during the procedure were ECG, oxygen saturation, and arterial blood pressures. Quantitative PA blood flow was measured by VEC MRI after the catheter was positioned in the main PA. Simultaneously, analogue invasive pressures were measured through the liquid filled catheter. All measurements (thermodilution and MRI) were repeated three times.

\section{Clinical study}

A total of 15 patients were studied. Of these, five patients (mean (SD) age 42 (11) years, mean (SD) body weight 65 (15) $\mathrm{kg}$ ) with a haemodynamically insignificant patent foramen ovale served as a control group and had cardiac catheterisation for routine closure of the foramen after prior occurrence of a paradoxical cerebral insult. The other 10 patients had PHT. Of these, five had primary PHT (mean (SD) age 17 (6) years, mean (SD) body weight $48(9) \mathrm{kg}$ ) and five had secondary PHT (mean (SD) age 13 (4) years, mean (SD) body weight $27(8) \mathrm{kg}$ ). Secondary PHT was caused by chronic pulmonary overcirculation in the presence of ventricular septal defects. All patients had a history of PHT for at least two years and had subsystemic PA pressures. At the time of the study, none of the patients were receiving selective pulmonary vasodilating agents. The study complied with the Declaration of Helsinki and was approved by an institutional review. Informed consent was obtained from all study participants or their guardians.

All patients were transferred for routine cardiac catheterisation into the cardiac catheterisation laboratory. Cardiovascular pressures were measured in the PA and LA and in patients with patent foramen ovale the foramen was closed by transcatheter implantation of an Amplatzer septal occluder. At the end of the cardiac catheterisation session all catheters were removed from the vascular system and the patients were transferred to the MRI unit. The catheterisation laboratory was located about $30 \mathrm{~m}$ from the MRI unit and was held available as a safety back up during all MRI procedures. A 6 French wedge catheter was inserted through a femoral vein sheath and advanced into the PA under magnetic resonance fluoroscopy. Analogue invasive PA pressures and VEC MRI derived quantitative blood flow were measured simultaneously. In patients with PHT, measurements were repeated after 10 minutes of inhaled nitric oxide. Patients were mildly sedated as clinically indicated. All measurements were repeated three times. LA pressures were measured in the cardiac catheterisation laboratory immediately before MRI but were not repeated during MRI. Vital signs continuously monitored during the procedure were ECG and arterial blood pressures, and blood gases were analysed.

\section{MRI techniques \\ MRI controlled catheter position monitoring}

The balloon of the wedge catheter was temporarily filled with carbon dioxide for susceptibility based catheter visualisation during magnetic resonance fluoroscopy. ${ }^{18}$ Magnetic resonance fluoroscopy was based on an interactive real time steady state free precession sequence. Imaging parameters were as follows: repetition time, $2.7 \mathrm{~ms}$; echo time, $1.4 \mathrm{~ms}$; flip angle, $55^{\circ}$; field of view, variable (200-350); reduced field of view, $80 \%$; matrix, $144 \times 144$; slice thickness, variable (8$10 \mathrm{~mm}) ;$ and $\mathrm{k}$-space filling, radial. The acquisition frame rate was 9 frames/s and the reconstruction and display rate was online (290 frames/s). No ECG gating was used for image acquisition.

\section{Pulmonary flow measurements}

Quantitative pulmonary blood flow was measured at the distal portion of the main PA by VEC MRI sequences with sensitivity encoding. ${ }^{19}$ Sequence parameters were as follows: repetition time, $14 \mathrm{~ms}$; echo time, $7 \mathrm{~ms}$; flow direction, through-plane; velocity encoding, $100-200 \mathrm{~cm} / \mathrm{s}$; slice thickness, $8 \mathrm{~mm}$; sensitivity encoding factor, 2; matrix, $256 \times 256$; field of view, variable (200-350); reduced field of view, 50\%; cardiac phase duration, $25 \mathrm{~ms}$; cardiac phases, variable (depending on heart rate with a mean of 27 (2) phases); and cardiac trigger was retrospective to the $\mathrm{R}$ wave.

\section{Measurement of cardiovascular pressures}

All pressure data were acquired with liquid filled catheters that were connected to a Statham transducer shielded by a copper mesh against radiofrequency pulses. The length of the pressure line was $1 \mathrm{~m}$. The pressure transducers were zero referenced at the mid chest level and cardiovascular pressures were measured at end expiration. Analogue signals were amplified (2 V/100 mm Hg output) and digitised with a sample rate of $250 \mathrm{~Hz}$ and 16 bit resolution by an analogue to digital conversion card (National Instruments, Houston, Texas, USA) connected to a personal computer. Measurements were recorded and analysed with custom software (C++ and MATLAB based software developed at our institution).

\section{Nitric oxide inhalation technique}

Nitric oxide was supplied from a source tank (400 ppm) and was delivered in $100 \%$ oxygen at $20 \mathrm{ppm}$ through a face mask. All haemodynamic variables were measured at steady state after 10 minutes of nitric oxide inhalation. The delivered dose of nitric oxide was two times the dose previously reported to offer a maximum pulmonary vasodilator effect in patients with primary PHT. ${ }^{20}$ Both nitric oxide and oxygen are considered potent vasodilators. ${ }^{20}$

\section{Calculations}

PVR (Wood units $\cdot \mathrm{m}^{2}$ ) was calculated from mean PA pressures ( $\mathrm{mm} \mathrm{Hg}$ ) divided by PA forward flow (l/min). Parameters were indexed to body surface area $\left(\mathrm{m}^{2}\right)$. LA pressures were not included in the calculation because they were at normal levels in all in vivo experiments and in all patients studied. By using VEC MRI, instantaneous pulmonary flow volumes were integrated and multiplied by heart rate to give PA forward and regurgitant blood flow (l/min). For thermodilution, right ventricular cardiac outputs were considered equivalent to PA forward flow in the absence of shunts or valvar insufficiency.

\section{Statistical analysis}

Normal distribution of data was tested with the KolmogorovSmirnov test. Data are presented as mean (SD) where appropriate. The variability of three repeated measurements was defined as inter-examination variability (measurement precision) and expressed as a percentage. All MRI measurements were analysed independently by two investigators.

\section{In vivo study}

Paired Student's $t$ test was used to compare PA pressures, flow, and resistance measured during conventional cardiac catheterisation and MRI. A value of $\mathrm{p}<0.05$ was considered 

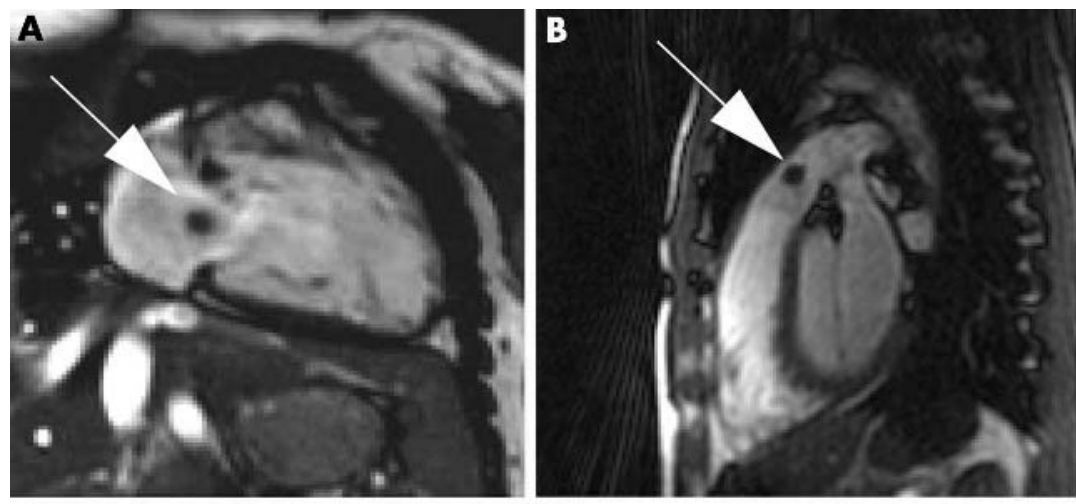

Figure 1 Magnetic resonance fluoroscopic images of the heart. Note the balloon tipped catheter (arrows) in the (A) right atrium, (B) right ventricular outflow tract, and (C) main pulmonary artery of a control patient, and (D) in the largely dilated main pulmonary artery of a patient with pulmonary hypertension (PHT). The balloon is inflated with carbon dioxide and produces a localised susceptibility artefact (arrows).
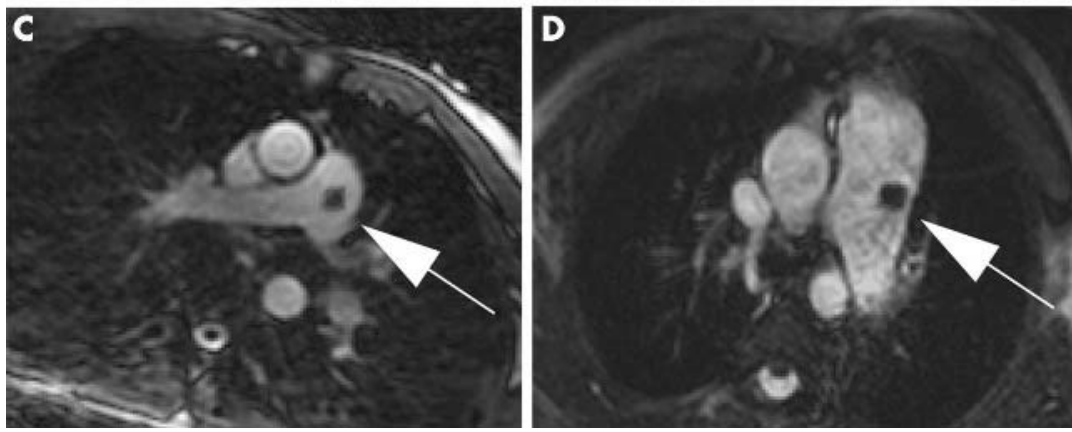

significant. Agreement between thermodilution and MRI derived PA flow volume and resistance was analysed by the Bland-Altman test.

\section{Clinical study}

Unpaired Student's $t$ test with Bonferroni correction for multiple analysis was used to compare PA pressures, flow, and resistance between the control patients and patients with PHT. Paired Student's $t$ test was used within the PHT group to determine the effects of nitric oxide inhalation on PA pressure, flow, and resistance. A value of $p<0.05$ was considered significant.

\section{RESULTS}

\section{MRI controlled catheter position monitoring}

Fast and reliable catheter control within the MRI environment was achieved in both in vivo and clinical experiments. The balloon tipped catheter was well visualised when the balloon was inflated with carbon dioxide (fig 1). The inflated balloon produced a circumscribed susceptibility artefact, which was clearly distinguishable from the bright blood pool of the right heart chambers and the main PA (fig l). Monitoring the advancement of the catheter from the vena cava to the PA required an average of four tomographic acquisitions. The needed scan duration was 6 (4) minutes.

\section{Haemodynamic in vivo data}

Cardiovascular pressure and global parameters of cardiovascular function

Tricuspid or pulmonary regurgitation or intracardiac shunts were not noted. LA pressure was 3.1 (0.8) mm Hg. Global parameters of cardiovascular function remained constant during the cardiac catheterisation and MRI sessions. Mean (SD) heart rate was 95 (12) beats/min and mean arterial blood pressure 63 (14) $\mathrm{mm} \mathrm{Hg}$. Heart rate and blood pressures changed less then $10 \%$ between the thermodilution and MRI studies.

No interference between the MRI pulses and the pressure transducer was noted. There was no significant difference between PA pressures derived in the catheterisation and MRI laboratories (table 1).

\section{Pulmonary flow volume}

No phase offset was noted for VEC MRI derived phasic blood flow measurements. There was no significant difference between thermodilution and VEC MRI derived PA flow volumes $(p=0.09)$. Bland-Altman test showed agreement between both methods (fig 2). All measurements were within the interval of $\pm 2 \mathrm{SD}$. The bias (MRI-thermodilution) was $-0.6 \mathrm{l} / \mathrm{min} / \mathrm{m}^{2}$. The standard deviation of difference values was $0.7 \mathrm{l} / \mathrm{min} / \mathrm{m}^{2}$. Inter-examination variability was high in

Table 1 In vivo haemodynamic data obtained by thermodilution and magnetic resonance imaging (MRI)

\begin{tabular}{|c|c|c|c|c|}
\hline & \multicolumn{2}{|c|}{ Thermodilution } & \multicolumn{2}{|l|}{ MRI } \\
\hline & Mean (SD) & Variability (\%) & Mean (SD) & Variability (\%) \\
\hline PA flow volume $\left(1 / \mathrm{min} / \mathrm{m}^{2}\right)$ & $3.3(0.8)$ & $7.8(3.1)$ & $2.8(0.3)$ & $2.4(0.5)$ \\
\hline Mean PA pressure $(\mathrm{mm} \mathrm{Hg})$ & $12.1(2.7)$ & $1.4(0.5)$ & $13.3(3.2)$ & $1.6(0.6)$ \\
\hline PA resistance (Wood units $\cdot \mathrm{m}^{2}$ ) & $0.9(0.5)$ & $6.2(2.2)$ & $1.1(0.3)$ & $2.1(0.3)$ \\
\hline
\end{tabular}

Data are the mean (SD) of three repeated measurements. Inter-examination variability was determined from three repeated measurements. PA, pulmonary artery. 


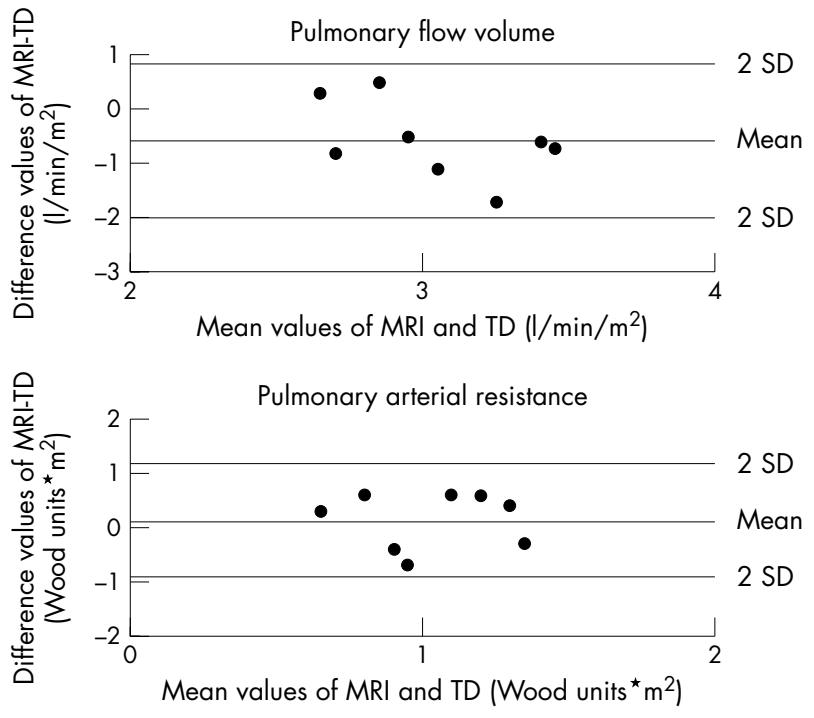

Figure 2 Bland-Altman plots for comparison of magnetic resonance imaging (MRI) and thermodilution (TD) derived pulmonary flow volumes and arterial resistance in vivo.

data obtained by the thermodilution technique but small in MRI data (table 1).

\section{Pulmonary vascular resistance}

There was no significant difference between thermodilution and MRI derived PVR ( $p=0.1$ ) (table 1). Bland-Altman test showed agreement between both methods (fig 2). All measurements were within the interval of \pm 2 SD. The mean of difference values (MRI-thermodilution) was 0.14 Wood units $\cdot \mathrm{m}^{2}$. The standard deviation of difference values was 0.52 Wood units $\cdot \mathrm{m}^{2}$. Inter-examination variability was high with the thermodilution technique but small with MRI (table 1).

\section{Haemodynamic clinical data}

\section{Cardiovascular pressures}

LA pressures were not increased in either patient group (3.4 (0.4) $\mathrm{mm} \mathrm{Hg}$ for controls and $3.8(0.6) \mathrm{mm} \mathrm{Hg}$ for patients with PHT). PA pressures were significantly increased in patients with PHT compared with the control group $(\mathrm{p}<0.001)$ (table 2$)$. There was no significant difference between PA pressures acquired in the conventional catheterisation and in the MRI laboratories $(\mathrm{p}=0.9)$ (table 2).

\section{PA flow volume}

No phase offset was noted for VEC MRI derived phasic blood flow measurements. Measurements showed no pulmonary regurgitation (retrograde flow) in either patient group. Compared with control, VEC MRI measured PA flow volumes were significantly smaller in patients with primary PHT $(p<0.01)$ but significantly larger in patients with secondary PHT and ventricular septal defect $(\mathrm{p}<0.01)$ (fig 3, table 2). Inter-examination variability of three repeated VEC MRI measurements was $2.8(0.6) \%$.

\section{Pulmonary vascular resistance}

MRI derived PVR was significantly increased in patients with PHT compared with the control group $(\mathrm{p}<0.001)$ (table 2$)$. In addition, PVR was significantly higher in patients with primary PHT than in patients with secondary forms of PHT $(\mathrm{p}<0.01)$ (table 2$)$. Inter-examination variability of three repeated MRI derived measurements was $2.4(0.5) \%$. There was no significant difference as determined for each patient group $(\mathrm{p}=0.4)$.

\section{Effects of nitric oxide inhalation}

During inhalation of nitric oxide mean pulmonary vascular pressure decreased significantly $(p<0.01)$ (fig 4$)$. At the same time, total pulmonary blood flow increased $(p<0.05)$ and in concordance PVR decreased $(p<0.05)$ (fig 4$)$. Interexamination variability of three repeated MRI derived measurements was $2.6(0.4) \%$.

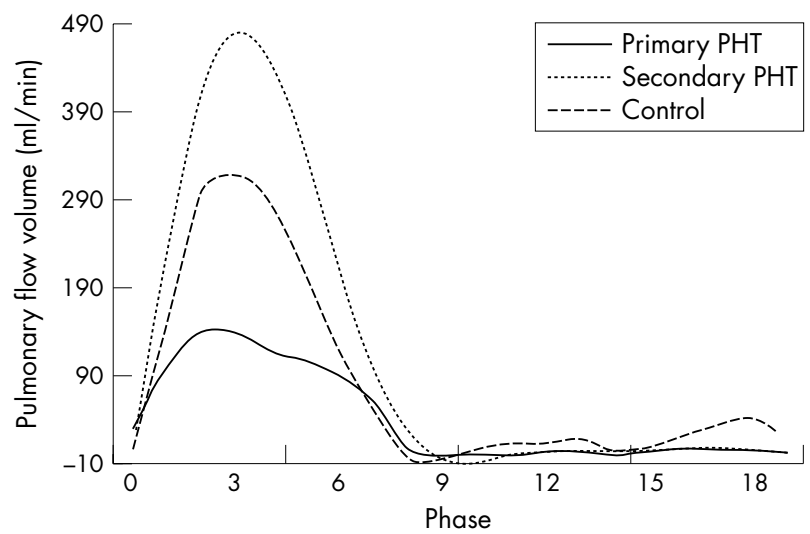

Figure 3 Representative phasic pulmonary flow as measured with velocity encoded cine MRI. Note differences in flow volumes between patients with primary and secondary forms of PHT.

Table 2 Patient characteristics and haemodynamic data measured during MRI

\begin{tabular}{|c|c|c|c|}
\hline & Control group & Primary PHT & Secondary PHT \\
\hline \multicolumn{4}{|l|}{ Patient characteristics } \\
\hline \multicolumn{4}{|l|}{ Heart rate (beats/min) } \\
\hline MRI laboratory & $65(11)$ & $73(9)$ & $82(8)$ \\
\hline Catheterisation laboratory & $63(9)$ & $72(10)$ & $82(9)$ \\
\hline \multicolumn{4}{|c|}{ Mean systemic arterial pressure $(\mathrm{mm} \mathrm{Hg})$} \\
\hline MRI laboratory & $73(8)$ & 71 (7) & $68(9)$ \\
\hline Catheterisation laboratory & $74(9)$ & 71 (12) & $69(8)$ \\
\hline \multicolumn{4}{|l|}{ Haemodynamic data } \\
\hline \multicolumn{4}{|l|}{ Mean PA pressure $(\mathrm{mm} \mathrm{Hg})$} \\
\hline MRI laboratory & $12.3(3.1)$ & $57.4(21.4)^{*}$ & $43.5(16.3)^{*}$ \\
\hline Catheterisation laboratory & $12.6(3.2)$ & $57.1(10.2)^{*}$ & $43.7(17.8)^{*}$ \\
\hline \multicolumn{4}{|l|}{ Measures during MRI } \\
\hline PA flow volume $\left(1 / \mathrm{min} / \mathrm{m}^{2}\right)$ & $3.1(0.3)$ & $2.2(0.6)^{*}$ & $3.7(0.9)^{*} \dagger$ \\
\hline Baseline PVR (Wood units $\mathrm{m}^{2}$ ) & $1.2(0.8)$ & $17.2(4.5)^{*}$ & $8.9(3.9)^{*} \dagger$ \\
\hline
\end{tabular}



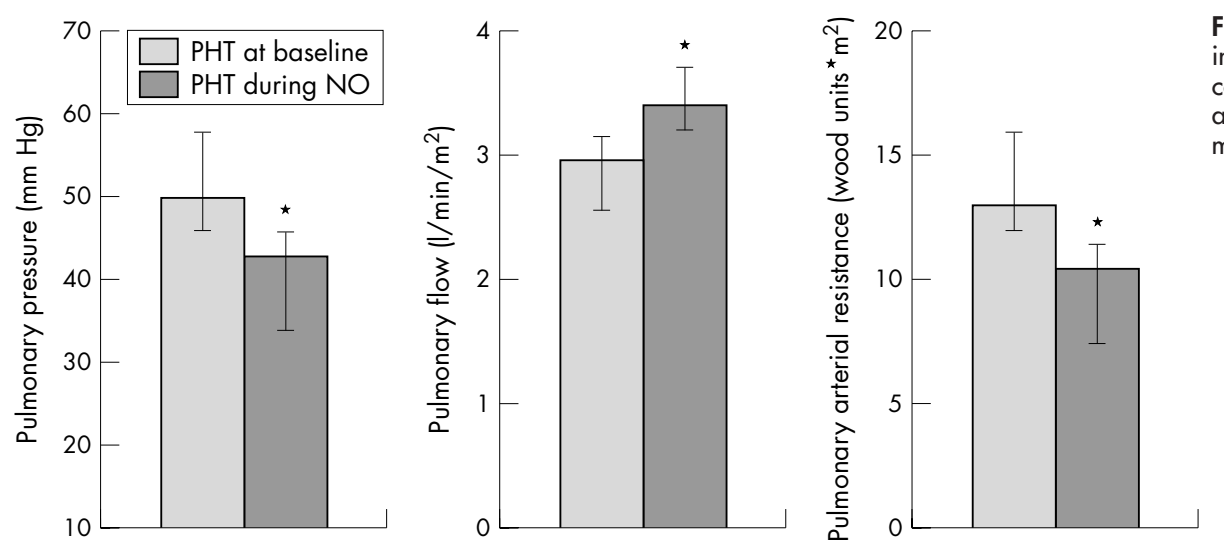

Figure 4 Effects of nitric oxide (NO) inhalation in eight patients with PHT compared with measurements acquired at baseline. Data are presented as mean (SD); ${ }^{\mathrm{p}}<0.05$.

\section{DISCUSSION}

In this study, assessment of invasive PA pressures combined with VEC MRI derived PA blood flow volumes provided detailed haemodynamic information about the pulmonary vascular system including PVR. Pressure was measured through flow directed catheters guided into the PA under magnetic resonance fluoroscopy. This MRI method was first validated in vivo and subsequently applied to patients with a broad range of PVR. The major findings of both components of this study are, firstly, that flow directed catheters can be guided into the PA under MRI control; secondly, that PVR can be measured with good precision with the proposed MRI technique; and, thirdly, that MRI seems to be a promising tool to assess PVR in patients with primary and secondary forms of PHT.

\section{MRI controlled catheterisation}

Over the past few years MRI has gained increasing acceptance for its ability to guide endovascular catheter based diagnostic or interventional procedures. MRI controlled stent placement and balloon dilatation of the PA were successful in the swine models. ${ }^{11}{ }^{21}$ More recently, Schala and colleagues $^{12}$ and Muthurangu and associates ${ }^{13}$ reported MRI controlled cardiac catheterisation in animal studies and in the first human studies. In these studies, a hybrid MRI $x$ ray system was used, in which catheters can be placed with $x$ ray fluoroscopy readily available as a back up.

In the current study MRI guided cardiac catheterisation was achieved with a susceptibility based passive catheter tracking method, which does not yield any potential patient hazards. ${ }^{22}$ The inflated balloon produced a circumscribed susceptibility artefact at the tip of the catheter, which was clearly distinguished from the bright blood pool of the heart and main PA.

In this study MRI guidance of catheters into the PA required an average time of only six minutes. No major difficulties where encountered when the technique was applied to simple anatomy as in this study. However, this passive catheter tracking modality may not generate sufficient contrast for reliable MRI guidance if used in more complex anatomy such as that found in a variety of congenital heart diseases. To perform such catheterisation procedures a catheter tracking modality is warranted that is safe for patients and allows for automated slice and tip detection. ${ }^{23}$ In addition, catheter tracking modalities should allow for visualisation of the catheter shaft to avoid looping or knotting of the catheter. Until final development of such catheter tracking methods, $x$ ray systems should be held available during MRI as a safety back up. This is of particular importance given that patients with PHT can develop hypertensive crises during endovascular procedures.

\section{Assessment of PA resistance}

PVR is measured or estimated with a variety of methods, which are often limited in terms of accuracy or measurement precision. Thermodilution and Fick techniques are in routine clinical use. However, both techniques have been shown to be associated with high measurement variability. ${ }^{4-7}$ In the preliminary in vivo study we found a high inter-examination variability of $6.2 \%$ for thermodilution technique and a low inter-examination variability of $2.1 \%$ for MRI derived PVR.

In contrast with previously published reports, ${ }^{13}$ we applied in this study the proposed MRI method to homogenous patient groups, including control patients with no major cardiovascular malformation or disease. In this control group, our resulting MRI derived PVR was in the expected range of 1-3 Wood units $\cdot \mathrm{m}^{2} .^{24}$ In the patients with primary and secondary forms of PHT, PVR was substantially increased but at subsystemic levels. PA pressures were not significantly higher in the patients with primary forms of PHT than in those with secondary forms. However, VEC MRI clearly showed pulmonary overcirculation in patients with ventricular septal defect caused by a left to right shunt of 1.3 (0.6) $\mathrm{ml} / \mathrm{m}^{2}$. Information about differential perfusion of the PA and intracardiac shunts as provided by MRI can be very valuable for clinical and surgical decision making.

Principal management of PHT has changed significantly over the past decade. Selective pulmonary vasodilatation may be achieved with recently approved pulmonary vasodilators, thus reducing right ventricular afterload, improving transpulmonary blood flow, and ultimately preventing lung or heart-lung transplantation or death. ${ }^{3}$ An accurate and reproducible method for monitoring PVR in such patients is desired. In the current study both baseline and MRI derived measurements during nitric oxide inhalation were highly precise. This result implies that the proposed MRI method is potentially suited to monitor the effects of selective PA vasodilating drugs.

In the current study MRI derived clinical PVR data were not further validated with an alternative method such as thermodilution or Fick because, firstly, our in vivo results clearly showed that MRI derived PVR inter-examination variability was lower than that of the thermodilution technique; secondly, previous studies have shown the superiority of VEC MRI over the Fick method for assessment of quantitative PA flow volumes ${ }^{17}$; and, thirdly, acceptable correlation and agreement between MRI and Fick derived PVR were reported for patients without or with mild PHT. ${ }^{13}$ 
Another method for assessment of PVR is the use of Doppler guidewires. ${ }^{9}$ However, there is concern that the small vascular regions, such as those sampled with guidewires, may not represent the entire PA vascular system. This limitation is of particular importance in patients with an inhomogeneous pulmonary vascular bed, such as patients after the Glenn or Fontan procedure. ${ }^{25} \mathrm{~A}$ study by Fratz and colleagues ${ }^{16}$ showed that differential left and right PA perfusion can be accurately assessed by VEC MRI. It would be desirable to measure both total and individual branch PVR in a broad variety of patients with congenital heart disease. The MRI technique proposed in this study may be potentially suited for such an investigation.

A variety of non-invasive MRI and ultrasound methods have been described for estimation of PA pressures and resistance. ${ }^{102627}$ Most of these methods are based on estimation of mean PA blood pressure from pressure wave velocity analysis. However, these non-invasive approaches are investigator dependant, require time consuming postprocessing of data, or have the drawback that multiple sources can induce errors. ${ }^{10} 27$

The accuracy of VEC MRI was determined excessively in vitro and in vivo. ${ }^{14} 28$ The method is known to have limitations in the presence of arrhythmia and turbulent flow caused by, for example, valvar or vascular obstruction. ${ }^{15}$ In the current study none of the patients had arrhythmias or signal jet voids caused by turbulent flow. However, VEC magnitude images showed largely dilated PA in the patients with PHT (fig 1). The mean cross sectional surface area changed between ejection and the end diastolic heart phase by $1.4(0.7) \mathrm{cm}^{2}$ when measured at the mid portion of the PA but by only $0.6(0.4) \mathrm{cm}^{2}$ when measured at the distal portion of the PA. To minimise the influence of PA Windkessel and capacitive function, all pressure and VEC MRI measurements were acquired at the distal portion of the main PA.

\section{Study limitation}

The temporal resolution of VEC MRI sequences is relatively low. A much higher temporal resolution is desired to account better for fast changes in flow, which would allow also for assessment of PA impedance at high frequencies. Recently proposed real time VEC MRI sequences are promising to overcome this limitation and should be investigated in future research. ${ }^{29}$ In the current study agreement of PVR as measured with the thermodilution technique and with MRI was assessed in animal experiments with low variation of haemodynamic conditions. However, in patients with PHT haemodynamic conditions are much more variable. This fact must be considered if future research is addressed in the clinical context.

In conclusion, magnetic resonance fluoroscopy is suited to guide flow directed catheters for measurement of invasive PA pressures. PVR can be precisely measured with the proposed MRI technique. Furthermore, the results indicate that MRI is a promising tool for measurement of PVR in patients with different degrees and forms of PHT.

\section{Authors' affiliations}

T Kuehne, S Yilmaz, I Schulze-Neick, P Ewert, P Lange, Department of Congenital Heart Diseases and Paediatric Cardiology, German Heart Institute, Berlin, Germany

E Wellnhofer, E Nagel, Department of Cardiology, German Heart Institute, Berlin

This study was funded in part by the Bundesmenisterium für Forschung und Bildung

\section{REFERENCES}

1 Friedman W, Heiferman M. Clinical problems of postoperative pulmonary vascular disease. Am J Cardiol 1982;50:631-6.

2 Spray T, Mallory G, Canter C, et al. Pediatric lung transplantation for pulmonary hypertension and congenital heart disease. Ann Thorac Surg 1992:54:216-23.

3 Schulze-Neick I, Hartenstein P, Li J, et al. Intravenous sildenafil is a potent pulmonary vasodilator in children with congenital heart disease. Circulation 2003; 108:11167-73.

4 Espersen K, Jensen E, Rosenborg D, et al. Comparison of cardiac output measurement techniques: thermodilution, Doppler, CO2-rebreathing and the direct Fick method. Acta Anaesthesiol Scand 1995;39:245-51.

5 Hillis L, Firth B, Winniford M. Variability of right-sided cardiac oxygen saturations in adults with and without left-to-right. Am J Cardiol 1986;58:129-32.

6 Van Grondelle A, Ditchey RV, Groves BM, et al. Thermodilution method overestimates low cardiac output in humans. Am J Physiol 1983;245:H690-2.

7 Wippermann C, Huth R, Schmidt F, et al. Continuous measurement of cardiac output by the Fick principle in infants and children: comparison with the thermodilution method. Intensive Care Med 1996;22:467-71.

8 Borges A, Wensel R, Opitz C, et al. Relationship between haemodynamics and morphology in pulmonary hypertension: a quantitative intravascular ultrasound study. Eur Heart J 1997; 18:1988-94.

9 Ivy D, Neish S, Knudson O, et al. Intravascular ultrasonic characteristics and vasoreactivity of the pulmonary vasculature in children with pulmonary hypertension. Am J Cardiol 1998;81:740-8.

10 Stevenson JG. Comparison of several noninvasive methods for estimation of pulmonary artery pressure. J Am Soc Echocardiogr 1989;2:157-71.

11 Kuehne T, Saeed M, Higgins CB, et al. Endovascular stents in pulmonary valve and artery in swine: feasibility study of MR imaging-guided deployment and postinterventional assessment. Radiology 2003;226:475-81.

12 Schala S, Saeed M, Higgins CB, et al. Magnetic resonance imaging guided cardiac catheterization in swine model of atrial septal defect. Circulation 2003:108:1865-70

13 Muthurangu V, Taylor A, Andriantsimiavona R, et al. Novel method of quantifying pulmonary vascular resistance by use of simultaneous invasive pressure monitoring and phase-contrast magnetic resonance flow. Circulation 2004; 110:826-34

14 Rebergen S, van der Wall E, Doornbos J, et al. Magnetic resonance measurement of velocity and flow: technique, validation, and cardiovascular applications. Am Heart J 1993;126:1439-56.

15 Stahlberg F, Sondergaard L, Thomsen C, et al. Quantification of complex flow using MR phase imaging--a study of parameters influencing the phase/ velocity relation. Magn Reson Imaging 1992;10:13-23.

16 Fratz S, Hess J, Schwaiger $M$, et al. More accurate quantification of pulmonary blood flow by magnetic resonance imaging than by lung perfusion scintigraphy in patients with Fontan circulation. Circulation 2002; 106:1510-3.

17 Beerbaum P, Korperich $H$, Barth $P$, et al. Noninvasive quantification of left-toright shunt in pediatric patients: phase-contrast cine magnetic resonance imaging compared with invasive oximetry. Circulation 2001;103:2476-82.

18 Miquel ME, Hegde S, Muthurangu V, ef al. Visualization and tracking of an inflatable balloon catheter using SSFP in a flow phantom and in the heart and great vessels of patients. Magn Reson Med 2004;51:988-95.

19 Beerbaum P, Korperich H, Gieseke J, et al. Rapid left-to-right shunt quantification in children by phase-contrast magnetic resonance imaging combined with sensitivity encoding (SENSE). Circulation 2003;108:1355-61.

20 Sitbon $\mathrm{O}$, Brenot F, Denjean A, et al. Inhaled nitric oxide as a screening vasodilator agent in primary pulmonary hypertension: a dose-response study and comparison with prostacyclin. Am J Respir Crit Care Med 1995; 151:384-9.

21 Rickers C, Seethamraju R, Jerosch-Herold M, et al. Magnetic resonance imaging guided cardiovascular interventions in congenital heart diseases. $J$ Interv Cardiol 2003; 16:143-7.

22 Bakker CJ, Bos C, Weinmann HJ. Passive tracking of catheters and guidewires by contrast-enhanced MR fluoroscopy. Magn Reson Med 2001;45:17-23.

23 Kuehne T, Fahrig R, Butts K. Pair of resonant fiducial markers for localization of endovascular catheters at all catheter orientations. J Magn Reson Imaging 2003;17:620-4.

24 Rudolph A. Congenital diseases of the heart: clinical-physiological considerations. New York: Futura Publishing Company, 2001.

25 Stevenson J. Effect of unilateral diaphragm paralysis on branch pulmonary artery flow. J Am Soc Echocardiogr 2002;15:1127-31.

26 Welch E, Duara S, Suguihara C, et al. Validation of cardiac output measurements with noninvasive Doppler echocardiography by thermodilution and Fick methods in newborn piglets. Biol Neonate 1994;66:137-45.

27 Laffon E, Laurent F, Bernard V, et al. Noninvasive assessment of pulmonary arterial hypertension by MR phase-mapping method. J Appl Physiol 2001; 90:2197-202

28 Evans A, Iwai F, Grist T, et al. Magnetic resonance imaging of blood flow with a phase subtraction technique: in vitro and in vivo validation. Invest Radiol 1993;28:109-15.

29 Thompson R, McVeigh E. Real-time volumetric flow measurements with complex-difference MRI. Magn Reson Med 2003;50:1248-55. 